\title{
Landau Level Spectroscopy of Electron-Electron Interactions in Graphene
}

\section{Citation}

Faugeras, C., S. Berciaud, P. Leszczynski, Y. Henni, K. Nogajewski, M. Orlita, T. Taniguchi, et al. 2015. "Landau Level Spectroscopy of Electron-Electron Interactions in Graphene." Physical Review Letters 114 (12) (March 27). doi:10.1103/physrevlett.114.126804.

\section{Published Version}

doi:10.1103/PhysRevLett.114.126804

\section{Permanent link}

http://nrs.harvard.edu/urn-3:HUL.InstRepos:30781093

\section{Terms of Use}

This article was downloaded from Harvard University's DASH repository, and is made available under the terms and conditions applicable to Other Posted Material, as set forth at http:// nrs.harvard.edu/urn-3:HUL.InstRepos:dash.current.terms-of-use\#LAA

\section{Share Your Story}

The Harvard community has made this article openly available.

Please share how this access benefits you. Submit a story.

Accessibility 


\title{
Landau Level Spectroscopy of Electron-Electron Interactions in Graphene
}

\author{
C. Faugeras, ${ }^{1}$ S. Berciaud, ${ }^{2}$ P. Leszczynski, ${ }^{1}$ Y. Henni, ${ }^{1}$ K. Nogajewski, ${ }^{1}$ M. Orlita, ${ }^{1}$ T. Taniguchi, ${ }^{3}$ K. Watanabe,${ }^{3}$ \\ C. Forsythe, ${ }^{4}$ P. Kim, ${ }^{4}$ R. Jalil, ${ }^{5}$ A. K. Geim, ${ }^{5}$ D. M. Basko, ${ }^{6, *}$ and M. Potemski ${ }^{1, \dagger}$ \\ ${ }^{1}$ Laboratoire National des Champs Magnétiques Intenses, CNRS, (UJF, UPS, INSA), BP 166, 38042 Grenoble, Cedex 9, France \\ ${ }^{2}$ Institut de Physique et Chimie des Matériaux de Strasbourg and NIE, UMR 7504, Université de Strasbourg and CNRS, \\ BP43, 67034 Strasbourg, Cedex 2, France \\ ${ }^{3}$ National Institute for Material Science, 1-1 Namiki, Tsukuba 305-0044, Japan \\ ${ }^{4}$ Department of Physics, Columbia University, New York, New York 10027, USA \\ ${ }^{5}$ School of Physics and Astronomy, University of Manchester, Manchester M13 9PL, United Kingdom \\ ${ }^{6}$ Université Grenoble 1/CNRS, Laboratoire de Physique et de Modélisation des Milieux Condensés (UMR 5493), \\ B.P. 166, 38042 Grenoble, Cedex 9, France
}

(Received 29 November 2014; published 27 March 2015)

\begin{abstract}
We present magneto-Raman scattering studies of electronic inter-Landau level excitations in quasineutral graphene samples with different strengths of Coulomb interaction. The band velocity associated with these excitations is found to depend on the dielectric environment, on the index of Landau level involved, and to vary as a function of the magnetic field. This contradicts the single-particle picture of noninteracting massless Dirac electrons but is accounted for by theory when the effect of electron-electron interaction is taken into account. Raman active, zero-momentum inter-Landau level excitations in graphene are sensitive to electron-electron interactions due to the nonapplicability of the Kohn theorem in this system, with a clearly nonparabolic dispersion relation.
\end{abstract}

DOI: 10.1103/PhysRevLett.114.126804

PACS numbers: 73.22.Pr, 73.43.Lp, 78.20.Ls

Single-particle electronic states in graphene follow the linear relativistic-type dispersion with the speed of light $c$ replaced by the band velocity $v \approx c / 300$. Recently, however, much attention was paid to modification of the dispersion relations and excitation spectra of quasiparticles induced by electron-electron interactions [1-3]. Indeed, pristine graphene can hardly be considered as a weakly interacting system [4-6]. The dimensionless interaction strength (ratio between typical Coulomb and kinetic energies), given by the fine-structure constant $\alpha \approx 1 / 137$ in quantum electrodynamics, is rescaled in graphene to $(c / v) \alpha \approx 2$. Screening (by a dielectric and/or conducting environment) modifies the Coulomb interaction strength in graphene, depending on its actual surrounding (substrate) and/or on the degree of departure from charge neutrality. In a uniform dielectric environment characterized by a dielectric constant $\varepsilon$, the effective fine-structure constant is $\alpha_{\varepsilon}=(c / v)(\alpha / \varepsilon)$. The renormalization of graphene bands by electron-electron interactions has mostly been studied without a magnetic field [3]. The latter drastically modifies the electronic spectrum by quantizing it into discrete and highly degenerate Landau levels (LLs). The inter-LL excitations, i.e., the electron excitations from filled to empty LL, are the collective electronic modes $[7,8]$ in any two-dimensional system, and their understanding is fundamental for a variety of quantum Hall effect phenomena [9]. The anticipated effects [10-14] of electron-electron interaction on LL spectrum in graphene have so far been little explored experimentally $[2,15]$.
In this Letter, we report on probing the inter-LL excitations, with magneto-Raman scattering experiments $[16,17]$, in three graphene systems with different dielectric environments. The measured spectrum of (Raman) inelastic light scattering accompanied by such electronic excitations provides information about their characteristic energies and evolution with the magnetic field. The noninteracting Dirac-like description of electrons fails to account for the full set of our experimental observations. An effective velocity associated with each inter-LL transition is not a single value but (i) depends on the dielectric environment, the departure from the noninteracting picture being most pronounced for suspended graphene, weaker for graphene encapsulated in hexagonal boron nitride, and rather small for graphene on graphite, (ii) varies logarithmically with the magnetic field, and (iii) is higher for transitions involving higher LLs. These observations can be qualitatively described in the Hartree-Fock approximation [10-12] or by the first-order perturbation theory (FOPT) in $\alpha_{\varepsilon}$ $[4,13]$. In particular, the absence of full cancellation between vertex and self-energy corrections implies violation of the Kohn theorem [18] for the Dirac spectrum. Notably, the vertex corrections invert the tendency of lowering the electron velocity with energy, resulting from the self-energy terms, which accounts for feature (iii); see also Refs. [2,15]. However, FOPT fails on the quantitative level when $\alpha_{\varepsilon}$ is not small. Beyond FOPT, the velocity renormalization can be addressed by the random-phase approximation [19] (see also Ref. [5]), which indeed better matches the experimental results. Under some additional 
assumptions, we estimate two relevant parameters, the band width and bare band velocity, which define the renormalized electronic dispersion.

Conventional absorption spectroscopy of inter-LL transitions in graphene [14] is restricted to far-infrared spectral range $(\lambda \sim 100 \mu \mathrm{m})$ and does not offer the necessary spatial resolution, otherwise required for probing small graphene flakes. Better resolution is offered by visible light techniques, such as Raman scattering, which is our method of choice. The possibility of observing Raman scattering from purely electronic, inter-LL excitations $[16,17,20]$ is a recent addition to the wide use of Raman scattering spectra of phonons for the characterization of different graphene structures [21,22]. We studied three distinct graphene systems: suspended graphene (G-S), graphene encapsulated in hexagonal boron nitride (G-BN), and graphene on graphite (G-Gr). G-S was suspended over a circular pit ( $8 \mu \mathrm{m}$ in diameter) patterned on the surface of a $\mathrm{Si} / \mathrm{SiO}_{2}$ substrate (see Refs. [17,23] for details of sample preparation). The G-BN structure consists of a graphene flake transferred onto a $\sim 50 \mathrm{~nm}$ thick layer of $\mathrm{hBN}$ and then covered by another hBN flake of the same thickness, all together placed on a $\mathrm{Si} / \mathrm{SiO}_{2}$ substrate (see Ref. [24] for details on a similar structure). The G-Gr flake was identified on the surface of freshly exfoliated natural graphite via mapping the Raman scattering response at a fixed magnetic field and searching for the position with the spectral features characteristic for graphene (see Refs. [25,26] for details of the procedure). The experimental arrangements (Refs. [16,17] and the Supplemental Material [27]) permitted Raman scattering experiments in magnetic fields up to $14 \mathrm{~T}$ (supplied by a superconducting coil, data collected for G-BN) or up to 29/30 T (supplied by a resistive magnet, data collected for G-S and G-Gr), at low temperatures $(4 \mathrm{~K})$ and with a spatial resolution of $\sim 1 \mu \mathrm{m}$ (diameter of the laser spot on the sample). Though the adequate electrical characterization of the investigated samples was not possible, we assume here that all our three graphene flakes are not far from being neutral systems; this is supported by many other studies of similar structures [23,24,28,29].

Besides the well-known spectral peaks due to phonons $[21,22,30]$, the magneto-Raman scattering spectra of each of our graphene samples show other, well-resolved peaks due to electronic inter-LL excitations, whose energies depend distinctly on the magnetic field. Those features are central for the present work. A collection of the related experimental data is presented in Fig. 1. Within the singleparticle approximation, the electronic dispersion in graphene is conical, $E(k)= \pm v k$. When a magnetic field $B$ is applied perpendicularly to the graphene plane, the continuous energy spectrum transforms into a series of discrete Landau levels $\left(L_{ \pm n}\right)$ with energies $E_{ \pm n}= \pm \sqrt{2 n} \hbar v / l_{B}$ (here $n=0,1,2, \ldots$, and $l_{B}=\sqrt{\hbar /(e B)}$ is the magnetic length); see upper inset to Fig. 1. We limit our

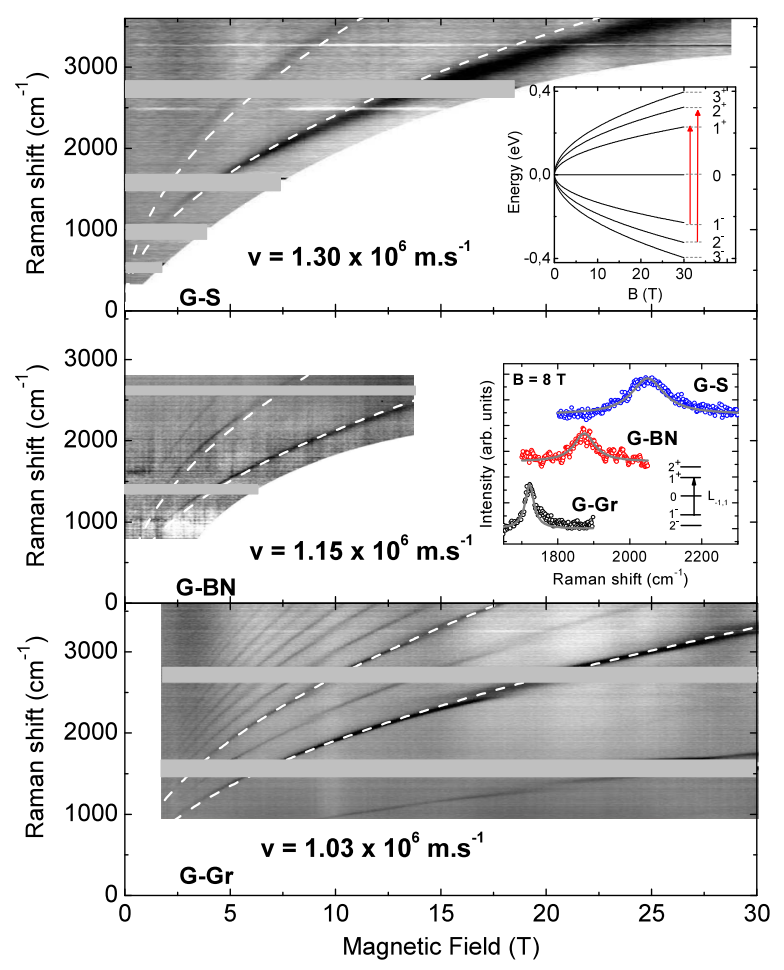

FIG. 1 (color online). Gray scale map of the electronic response in the magneto-Raman scattering spectra as a function of the magnetic field $(B)$ for suspended graphene (G-S, top panel), graphene encapsulated in $\mathrm{hBN}$ (G-BN, middle panel), and graphene on graphite (G-Gr, bottom panel). Each map represents the collection of differential spectra (the $B=0$ spectrum has been subtracted from each spectrum measured in a magnetic field $B$ ). Dashed white lines account for the $B$ evolutions of the two most pronounced inter-LL excitations, expected within a singleparticle approach, when assuming a $B$-independent but different for each sample band velocity $v$. Upper inset: scheme of the Landau level fan chart in graphene; two, $L_{-1,1}$ and $L_{-2,2}$, interLandau level excitations, well visible in the experiments on all samples, are indicated by red arrows. Middle panel inset: characteristic spectra due to $L_{-1,1}$ excitations measured at $B=8 \mathrm{~T}$ for $\mathrm{G}-\mathrm{Gr}$ (black symbols), G-BN (red symbols), and G-S (blue symbols). Gray curves are Lorentzian functions. Light gray horizontal ribbons on gray scale maps are to mask the (residual) contribution to Raman scattering due to phonons of graphene or graphite at $\sim 1600$ and $\sim 2700 \mathrm{~cm}^{-1}$ as well as of the Si substrate $\left(\sim 520\right.$ and $\sim 1000 \mathrm{~cm}^{-1}$ of $\mathrm{hBN}$ at $\sim 1370 \mathrm{~cm}^{-1}$.

considerations to the so-called symmetric $L_{-n} \rightarrow L_{n}$ $\left(L_{-n, n}\right)$ inter-LL transitions, which are expected to dominate the electronic Raman scattering response of graphene [20], and appear at energies $\hbar \omega_{-n, n}=2 E_{n}$, approximately. Tracing the $\hbar \omega_{-n, n}(B)$ dependencies on the top of the gray scale maps presented in Fig. 1, we recognize two transitions in the spectra of both G-S and G-BN, $L_{-1,1}$ and $L_{-2,2}$. G-Gr shows much richer spectra: a larger number of symmetric $L_{-n, n}$ transitions (at least up to $n=5$ ) as well as other, asymmetric, $\Delta|n|=1$ transitions. These latter transitions were predicted to be weakly allowed [20] but are 
nevertheless well seen in G-Gr, surprisingly, in a wide range of magnetic fields $[25,26]$. Notably the asymmetric transitions effectively couple to the $E_{2 g}$ phonon of graphene and are also visible in two other samples but only in the close vicinity of the phonon peak. Aiming at a systematic study of the spectra in different dielectric environments, we focus on the $L_{-1,1}$ and $L_{-2,2}$, which are clearly seen in all three cases in a wide range of the magnetic field. In all our samples, the $L_{-1,1}$ transition starts to be visible at magnetic fields as low as $\sim 2.5 \mathrm{~T}$, at energies $E_{\text {ons }} \sim 1000 \mathrm{~cm}^{-1}$. This observation defines the upper bound for the Fermi energy, $E_{F}<E_{\text {ons }} / 2 \simeq 60 \mathrm{meV}$, and confirms a relatively low doping in the studied graphene structures.

From the inspection of the $\hbar \omega_{-n, n}(B)$ traces drawn in Fig. 1, we easily identify the measured transitions, but at the same time notice some inconsistencies. First, using such an approximate data modeling, we are forced to use different velocities for each of our graphene samples: $v$ is set to $1.30 \times 10^{6}, 1.15 \times 10^{6}$, and $1.03 \times 10^{6} \mathrm{~m} / \mathrm{s}$ for G-S, G-BN, and G-Gr, respectively. Different mean velocities for each graphene specimen are directly visualized in the lower inset to Fig. 1: at fixed $B$ but for different samples the $L_{-1,1}$ transitions appear at clearly distinct energies. Moreover, the above $v$ parameters can only be considered as the mean velocity values, averaged over different transitions and over the range of magnetic fields applied; note, e.g., rather pronounced deviations between the white traces and the central peak positions for G-S (top panel of Fig. 1).

The shortcomings of the above data modeling are emphasized in Fig. 2, the central figure of this Letter. Data points (symbols) in this figure represent the velocity parameter that we associate with each observed transition and at each value of the magnetic field applied: $v_{n}^{\text {expt }}=$ $\omega_{-n, n}^{\text {expt }} l_{B} / \sqrt{8 n}$, where $\hbar \omega_{-n, n}^{\text {expt }}$ are the measured transition energies (central positions of the Raman scattering peaks). In the noninteracting case, all these velocities should collapse onto one single value. The extracted velocities $v_{n}^{\text {expt }}$, for different $L_{-n, n}$ transitions and for our three graphene structures, are plotted in Fig. 2 as functions of $\ln \left(l_{B_{0}} / l_{B}\right)=\ln \sqrt{B / B_{0}}$, where the reference magnetic field has been arbitrarily set to $B_{0}=1 \mathrm{~T}$. Each set of $v_{n}^{\text {expt }}$ versus $\ln \left(l_{B_{0}} / l_{B}\right)$ data can be fairly approximated by a linear function. The $v_{n}^{\text {expt }}$ traces are parallel within a given graphene structure but show different slopes for different samples. These features point towards the effects of renormalization of the electronic velocity and of energies of inter-LL transitions by the electron-electron interaction.

For neutral graphene at $B=0$, the FOPT in $\alpha_{\varepsilon}=\left(c / v_{0}\right)(\alpha / \varepsilon)$ gives the correction to the velocity $v$ [4],

$$
\frac{v}{v_{0}}=1-\frac{\alpha \varepsilon}{4} \ln \frac{|E|}{W}, \quad v=v_{0}-\frac{\alpha c}{4 \varepsilon} \ln \frac{|E|}{W},
$$

which depends on the electron energy $E$, counted from the Dirac point. Here, $v_{0}$ is the bare velocity, and $W$ is the

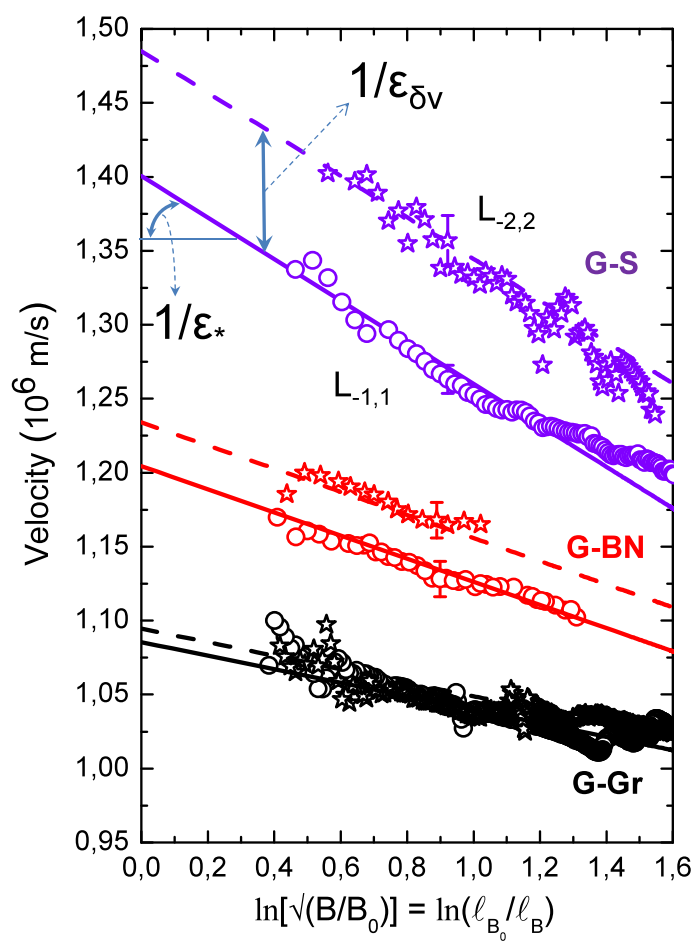

FIG. 2 (color online). Magnetic field dependence $\left(B_{0}=1 \mathrm{~T}\right)$ of the velocities associated with $L_{-1,1}$ and $L_{-2,2}$ inter-Landau level excitations shown with, correspondingly, open circles (solid lines) and open stars (dashed lines), as derived from the experiment (data modeling), for G-S, G-hBN, and G-Gr specimens. Straight lines follow Eq. (4) [see also Eq. (2)], with $\varepsilon_{*}=3.9,7.0$, and 12 for G-S, G-hBN, and G-Gr species, respectively, and the corresponding values for $\varepsilon_{\delta v}=1.3,3.7$, and 12 .

high-energy cutoff, which is of the order of the electronic bandwidth (a few $\mathrm{eV}$ ). The dielectric constant $\varepsilon$ is that of the surrounding medium for the suspended and encapsulated graphene. In a magnetic field, the FOPT calculation of the correction to the transition energy $\hbar \omega_{-n, n}$, performed analogously to that of Ref. [13], gives the following correction to the velocity (see the Supplemental Material [27]):

$$
v_{n} \equiv \frac{\omega_{-n, n} l_{B}}{\sqrt{8 n}}=v_{0}+\frac{\alpha c}{4 \varepsilon}\left(\mathcal{L}-\ln \frac{l_{B_{0}}}{l_{B}}\right)+\frac{\alpha c}{4 \varepsilon} C_{n},
$$

where $\mathcal{L}=\ln \left(W l_{B_{0}} / \hbar v_{0}\right)$ is a constant resulting from our choice of $l_{B_{0}}$ to set the horizontal scale in Fig. 2, and the numerical coefficients are $C_{1}=-0.398$ and $C_{2}=-0.197$. The coefficient in front of the logarithm, which determines the slopes in Fig. 2, is the same as in Eq. (1). This is because the logarithmic part originates from self-energy corrections due to interaction with states deep in the valence band, which are not sensitive to the Landau quantization and can be taken as $n$ independent. The constants $C_{n}$ include (i) the residual part of the selfenergies, resulting from interaction with states near the 
Fermi level, for which the level discreteness is important, and (ii) the vertex corrections (excitonic effects) [13].

Equation (2) accounts qualitatively for the main experimental trends seen in Fig. 2. For each sample $(\varepsilon)$, the dependencies of $v_{n}^{\text {expt }}$ versus $\ln \left(l_{B_{0}} / l_{B}\right)$ represent a set of parallel lines. The slope of these lines $[\propto 1 / \varepsilon$ according to Eq. (2)] correlates with the progressive increase of screening when shifting from G-S $(\varepsilon=1)$ to G-BN $\left(\varepsilon_{\mathrm{hBN}} \approx 5\right)$ and to G-Gr where screening by the conducting substrate can be viewed as a large effective $\varepsilon$. Notably, Eq. (2) also predicts $v_{2}>v_{1}$ for the same values of $\varepsilon$ and $B$ (as $C_{2}>C_{1}$ ), which would be the opposite if one simply substituted $E \rightarrow E_{n}^{0}$ in Eq. (1). This is due to vertex corrections. According to Eq. (2), $\delta v_{21} \equiv v_{2}-v_{1}=$ $(\alpha c / 4 \varepsilon)\left(C_{2}-C_{1}\right)$, which also agrees with the trend seen in Fig. 2, the decrease of $\delta v_{21}^{\text {expt }}$ with increasing $\varepsilon$.

However, Eqs. (1) and (2) fail to reproduce quantitatively the data shown in Fig. 2. This is with regard to both the apparent amplitude of the slopes of $v_{n}^{\text {expt }} \propto \ln (B)$ dependencies as well as the observed values of the relative shift $\delta v_{21}^{\text {expt }}$ between the velocities associated with $L_{1}$ and $L_{2}$ Landau levels.

If one used Eq. (2) with some adjustable $\varepsilon_{*}$ (effective dielectric constant) instead of $\varepsilon$, the slopes in Fig. 2 would correspond respectively to $\varepsilon_{*}=3.9 \pm 0.3,7.0 \pm 0.5$, and $12.0 \pm 1.0$ for G-S, G-BN, and G-Gr, quite different from the known $\varepsilon=1$ and 5 for G-S and G-BN. This is not very surprising, as the perturbative Eq. (2) does not have to work when the expansion parameter $\alpha_{\varepsilon}$ (exceeding 2 for $\varepsilon=1$ ) is not small. Fortunately, graphene offers another expansion parameter that can control the perturbation theory even when $\alpha_{\varepsilon} \sim 1$. This parameter is identified as $1 / N$, where $N$ is the number of electronic species, $N=4$ for graphene (the combined spin and valley degeneracy). In the $1 / N$ expansion, an infinite number of terms of the perturbation theory is resummed to all orders in $\alpha_{\varepsilon}$, selecting only those corresponding to the leading order in $1 / N$ [19]. The resulting series is equivalent to random-phase approximation, and it was explicitly shown that the subleading contribution is indeed small [5].

For the velocity renormalization at $B=0$, the $1 / N$ expansion boils down to the modification (depending on $\alpha$ ) of the coefficient in front of the logarithm in Eq. (1) [19]. For moderate values of $\alpha<2.5$, typical for graphene, this modified coefficient can be well approximated (with $1 \%$ precision) by [31]

$$
\frac{v_{0} \alpha_{\varepsilon}}{4} \rightarrow \frac{v_{0} \alpha_{\varepsilon}}{4\left(1+1.28 \alpha_{\varepsilon}\right)}=\frac{\alpha c}{4\left(\varepsilon+1.28 \alpha c / v_{0}\right)} .
$$

The above result can be seen as the added screening capacity $\varepsilon \rightarrow \varepsilon_{1 / N}=\varepsilon+1.28 \alpha c / v_{0}$ by the graphene Dirac electrons themselves. Assuming $v_{0}=0.88 \times$ $10^{6} \mathrm{~m} / \mathrm{s}$ (see below), we obtain $\varepsilon_{1 / N}=4.16$ for $\varepsilon=1$ (G-S) and 8.16 for $\varepsilon=5$ (G-BN), which are quite close to the measured values of $\varepsilon_{*}$. A large $\varepsilon_{*}=12$ $\left(\varepsilon_{1 / N} \simeq \varepsilon\right)$ value found for $\mathrm{G}-\mathrm{Gr}$ must effectively account for efficient screening by the conducting graphite substrate.

At this point, we apprehend the slopes of the lines in Fig. 2. The apparent amplitude of the velocity shifts $\delta v_{21}^{\text {expt }}$ remains to be analyzed. The measured values are $\delta v_{21}^{\text {expt }} \simeq\{0.084,0.029\} \times 10^{6} \mathrm{~m} / \mathrm{s}$ for G-S and G-BN, respectively, and we estimate that $\delta v_{21}^{\text {expt }} \leq 0.01 \times 10^{6}$ for G-Gr. On the other hand, Eq. (2) gives $\delta v_{21}=$ $(\alpha c / 4 \varepsilon)\left(C_{2}-C_{1}\right)=\{0.110,0.022,0.009\} \times 10^{6} \mathrm{~m} / \mathrm{s}$ for $\varepsilon=1,5$, and 12 , respectively, for G-S, G-BN, and G-Gr. The replacement $\varepsilon \rightarrow \varepsilon_{1 / N}$ in Eq. (2) results in an even worse agreement with the experiment. Indeed, this replacement is valid only for the leading logarithmic term, while the sublogarithmic terms should be calculated explicitly, and the simple combination $C_{n} / \varepsilon$ will be replaced, generally speaking, by some more complicated one.

In order to describe the whole set of experimental data, we assume the following ansatz:

$$
v_{n}=v_{0}+\frac{\alpha c}{4 \varepsilon_{*}}\left(\mathcal{L}-\ln \frac{l_{B_{0}}}{l_{B}}\right)+\frac{\alpha c}{4 \varepsilon_{\delta v}} C_{n},
$$

where $\varepsilon_{*}=3.9,7,12$ at the leading logarithmic term is in reasonable agreement with the $1 / N$ expansion. In the sublogarithmic term we fixed $C_{n}$ to be the same as in Eq. (2) and $\varepsilon_{\delta v}$ to depend only on $\varepsilon$ (but not on $n$ ). We do not have a proper theoretical justification for this assumption but in adopting it and setting $\varepsilon_{\delta v}=1.3,3.7$, 12 in order to reproduce the experimentally observed $\delta v_{21}$ we are left with only two adjustable parameters, $v_{0}$ and $\mathcal{L}$. Their best matching values are $v_{0}=0.88 \times 10^{6} \mathrm{~m} / \mathrm{s}$ and $\mathcal{L}=4.9$, i.e., $W=\left(\hbar v_{0} / l_{B_{0}}\right) e^{\mathcal{L}}=3.1 \mathrm{eV}$, in fair agreement with the bare velocity and the characteristic bandwidth expected in graphene [32].

Concluding, using micro-magneto-Raman scattering spectroscopy, we have studied inter-Landau level excitations in graphene structures, embedded in different dielectric environments. Understanding the energies of inter-LL excitations clearly falls beyond the single-particle approach (which refers to a simple Dirac equation) but appears to be sound when the effects of electron-electron interactions are taken into account. We confirm that the electronic properties of graphene on insulating substrates (weak dielectric screening) are strongly affected by electron-electron interactions, whereas conducting substrates favor the single-particle behavior (graphene on graphite studied here, but likely also graphene on metals [33-35] and graphene on $\mathrm{SiC}[36,37])$.

We thank Ivan Breslavetz, R. Bernard, S. Siegwald, and H. Majjad for technical support and P. Hawrylak for valuable discussions. This work was supported by the European Research Council, EU Graphene Flagship, and the Agence nationale de la recherche (Grant No. QuanDoGra 12 JS10-001-01). 
*denis.basko@1pmmc.cnrs.fr

†marek.potemski@lncmi.cnrs.fr

[1] D. C. Elias, R. V. Gorbachev, A. S. Mayorov, S. V. Morozov, A. A. Zhukov, P. Blake, L. A. Ponomarenko, I. V. Grigorieva, K. S. Novoselov, F. Guinea, and A. K. Geim, Nat. Phys. 7, 701 (2011).

[2] Z.-G. Chen, Z. Shi, W. Yang, X. Lu, Y. Lai, H. Yan, F. Wang, G. Zhang, and Z. Li, Nat. Commun. 5, 4461 (2014).

[3] D. N. Basov, M. M. Fogler, A. Lanzara, F. Wang, and Y. Zhang, Rev. Mod. Phys. 86, 959 (2014).

[4] J. González, F. Guinea, and M. A. H. Vozmediano, Mod. Phys. Lett. B 07, 1593 (1993).

[5] J. Hofmann, E. Barnes, and S. Das Sarma, Phys. Rev. Lett. 113, 105502 (2014).

[6] V. N. Kotov, B. Uchoa, V. M. Pereira, F. Guinea, and A. H. Castro Neto, Rev. Mod. Phys. 84, 1067 (2012).

[7] Yu. A. Bychkov, S. V. Iordanskii, and G. M. Eliashberg, Pis'ma Zh. Eksp. Teor. Fiz. 33, 152 (1981) [JETP Lett. 33, 143 (1981)].

[8] C. Kallin and B. Halperin, Phys. Rev. B 30, 5655 (1984).

[9] S. Das Sarma and A. Pinczuk, Perspectives in Quantum Hall Effects (Wiley, New York, 1997).

[10] A. Iyengar, J. Wang, H. A. Fertig, and L. Brey, Phys. Rev. B 75, 125430 (2007).

[11] Y. A. Bychkov and G. Martinez, Phys. Rev. B 77, 125417 (2008).

[12] Y. E. Lozovik and A. A. Sokolik, Nanoscale Res. Lett. 7, 134 (2012).

[13] K. Shizuya, Phys. Rev. B 81, 075407 (2010).

[14] M. Orlita and M. Potemski, Semicond. Sci. Technol. 25, 063001 (2010).

[15] Z. Jiang, E. A. Henriksen, L. C. Tung, Y.-J. Wang, M. E. Schwartz, M. Y. Han, P. Kim, and H. L. Stormer, Phys. Rev. Lett. 98, 197403 (2007).

[16] C. Faugeras, M. Amado, P. Kossacki, M. Orlita, M. Kühne, A. A. L. Nicolet, Y. I. Latyshev, and M. Potemski, Phys. Rev. Lett. 107, 036807 (2011).

[17] S. Berciaud, M. Potemski, and C. Faugeras, Nano Lett. 14, 4548 (2014)

[18] W. Kohn, Phys. Rev. 123, 1242 (1961).

[19] J. González, F. Guinea, and M. A. H. Vozmediano, Phys. Rev. B 59, R2474 (1999).

[20] O. Kashuba and V. I. Fal'ko, Phys. Rev. B 80, 241404 (2009).

[21] A. C. Ferrari and D. M. Basko, Nat. Nanotechnol. 8, 235 (2013).
[22] L. Malard, M. Pimenta, G. Dresselhaus, and M. Dresselhaus, Phys. Rep. 473, 51 (2009).

[23] S. Berciaud, S. Ryu, L. E. Brus, and T. F. Heinz, Nano Lett. 9, 346 (2009).

[24] C. R. Dean, A. F. Young, I. Meric, C. Lee, L. Wang, S. Sorgenfrei, K. Watanabe, T. Taniguchi, P. Kim, K. L. Shepard, and J. Hone, Nat. Nanotechnol. 5, 722 (2010).

[25] M. Kühne, C. Faugeras, P. Kossacki, A. A. L. Nicolet, M. Orlita, Y. I. Latyshev, and M. Potemski, Phys. Rev. B 85, 195406 (2012).

[26] C. Faugeras, J. Binder, A. A. L. Nicolet, P. Leszczynski, P. Kossacki, A. Wysmolek, M. Orlita, and M. Potemski, Europhys. Lett. 108, 27011 (2014).

[27] See the Supplemental Material at http://link.aps.org/ supplemental/10.1103/PhysRevLett.114.126804 for details of the experimental arrangements and of the calculation of the coefficients $C_{n}$.

[28] K. I. Bolotin, K. J. Sikes, Z. Jiang, M. Klima, G. Fudenberg, J. Hone, P. Kim, and H. L. Stormer, Solid State Commun. 146, 351 (2008).

[29] P. Neugebauer, M. Orlita, C. Faugeras, A.-L. Barra, and M. Potemski, Phys. Rev. Lett. 103, 136403 (2009).

[30] A. C. Ferrari, J. C. Meyer, V. Scardaci, C. Casiraghi, M. Lazzeri, F. Mauri, S. Piscanec, D. Jiang, K. S. Novoselov, S. Roth, and A. K. Geim, Phys. Rev. Lett. 97, 187401 (2006).

[31] Following the $1 / N$ expansion [19], the exact expression for the replacement of the coefficient in front of the logarithm in Eq. (1) is $\left(\alpha_{\varepsilon} / 4\right) \rightarrow\left(2 / \pi^{2}\right)\left\{1-\left(1 / \alpha_{\varepsilon}\right)+\left(2 / \pi \alpha_{\varepsilon}\right)+\right.$ $\left.\left[\arccos \left(\pi \alpha_{\varepsilon} / 2\right)\right] /\left[\sqrt{1-\left(\pi \alpha_{\varepsilon} / 2\right)^{2}}\right]\right\}$.

[32] R. Gillen and J. Robertson, Phys. Rev. B 82, 125406 (2010).

[33] K. S. Kim, Y. Zhao, H. Jang, S. Y. Lee, J. M. Kim, K. S. Kim, J.-H. Ahn, P. Kim, J.-Y. Choi, and B. H. Hong, Nature (London) 457, 706 (2009).

[34] J. Coraux, A. T. N'Diaye, C. Busse, and T. Michely, Nano Lett. 8, 565 (2008).

[35] X. Li, W. Cai, J. An, S. Kim, J. Nah, D. Yang, R. Piner, A. Velamakanni, I. Jung, E. Tutuc, S. K. Banerjee, L. Colombo, and R. S. Ruoff, Science 324, 1312 (2009).

[36] M. L. Sadowski, G. Martinez, M. Potemski, C. Berger, and W. A. de Heer, Phys. Rev. Lett. 97, 266405 (2006).

[37] M. Orlita, C. Faugeras, P. Plochocka, P. Neugebauer, G. Martinez, D. K. Maude, A.-L. Barra, M. Sprinkle, C. Berger, W. A. de Heer, and M. Potemski, Phys. Rev. Lett. 101, 267601 (2008). 